\title{
THE THEORY OF FUNCTIONS.
}

Introduction to the Theory of Analytic Functions. By J. HARKNess and F. Morley. London, Macmillan and Co., 1898. $8 \mathrm{vo}, \mathrm{xv}+336 \mathrm{pp}$.

The authors, already well known through their Treatise on the theory of functions, have laid the mathematical public under a new obligation by their "Introduction to the theory of analytic functions." The name which the authors have chosen for their new book gives only an inadequate idea of its scope and object; on the one hand it is not an introduction in the sense of a first introduction to the theory of functions ; on the other hand it is much more than an introduction ; it may be shortly described as a very complete treatise on Weierstrass's general theory of functions with applications to elliptic and algebraic functions (Chapters VI., XIX., XXI.), preceded by an introduction devoted to the number concept' and the geometric interpretation of complex quantities (Chapters I.-V.), and followed by a short account of some of the leading ideas of Riemann and Cauchy (Chapters XX., XXII.).

\section{The Introductory and Concluding Chapters.}

The book begins with a sketch of the system of real numbers considered as ordinal numbers (Chapter I.). A row of objects is considered with regard to their order, say from left to right. To count the objects means to label them, not primarily to say how many there are; and the integers are, primarily, mere labels. The object after which we begin to count, is labelled 0 ; if there are also objects to the left of it, they are labelled $-1,-2$, etc. Fractions are introduced by a process of relabelling : Pick out of the original row the objects $p, 2 p, 3 p \cdots$, and relabel them $1,2,3, \cdots$; if $\mu$ is not divisible by $p$, the object originally labelled $\mu$ is relabelled $\frac{\mu}{p}$. At the same time rules are given to decide whether two rational numbers are equal, and if not, which is the greater. A different kind of relabelling, viz., a change of origin, is used to define addition without reference to quantity. Also special irrationals like $\sqrt{2}$ can be introduced by a similar process of relabelling; but for the general definition of irrationals Dedekind's idea of a cut in 
the totality of rational numbers is adopted and it is shown how every decimal, finite or infinite, defines a cut.

Chapter II. gives the geometric representation of complex numbers and of the elementary algebraic operations with them, with numerous applications to geometrical problems. Along with the usual representation of a complex number by a point or by a stroke, the interpretation as an operator is emphasized, and, as in quaternions, the consideration of the quotient precedes that of the product.

Chapter III. contains a detailed study of the bilinear transformation interpreted as a one-to-one correspondence between two planes. The starting point is the geometric interpretation of the two equations

$$
a m\left(\frac{x-x_{1}}{x-x_{2}}\right)=\alpha \text { and }\left|\frac{x-x_{1}}{x-x_{2}}\right|=\rho ;
$$

the former represents an arc of a circle through $x_{1}, x_{2}$, the latter a complete circle "about $x_{1}, x_{2}$ ", that is a circle with respect to which $x_{1}, x_{2}$ are inverse points; the two circles are orthogonal.

By this means a very elegant treatment of the general bilinear transformation is obtained; and at the same time a simple geometric interpretation of two harmonic pairs.

Further the anharmonic ratio of four points and the isogonality of the transformation are considered and finally the subject is connected with the theory of absolute inversion in space. An interesting-apparently new-theorem is given, viz., that every bilinear transformation between two planes is equivalent to two inversions in space.

In Chapter IV. "Geometric theory of the logarithm and exponential" the authors intend to give an elementary geometric treatment of $\log \xi$, analogous to the way in which $\sin \xi$ is treated in trigonometry. For this purpose, the existence of the equiangular spiral is assumed, "which may be constructed by placing a right cone with axis vertical and attaching a thread $A P$ to a point $P$ of it. The point $P$ is held in the horizontal plane through the vertex $O$; the thread is then wound round the cone, without being allowed to slip. The curve described by the point $P$ in its plane is the spiral in question, and it can be proved in an elementary way that it cuts all rays from $O$ at a constant angle. Choose this angle to be $\pi / 4$; and then measuring the angle $\theta$ from the point where $O P=\rho$ has the value 1 , define

$$
\theta=\log \rho_{.}, "
$$


It follows then easily that

$$
\begin{gathered}
D \log \rho=\frac{1}{\rho} \\
\log \frac{\rho_{1}}{\rho}=\log \rho_{1}-\log \rho .
\end{gathered}
$$

The logarithm of a complex quantity $x_{1}$ is defined by

$$
\int_{1}^{x_{1}} \frac{d x}{x}
$$

and the mapping by means of $\log x$ is discussed with application to Mercator's projection.

The exponential is defined as the inverse of the logarithm. The mapping by means of $x=\exp y$ leads in a natural way to the "Napierian motion" defined by $x_{t}=x_{0} \exp b t$, which plays an important part in the following Chapter V., in which the bilinear transformation is taken up again and interpreted as a transformation of a plane into itself, produced by a motion of all the points of the plane.

The same Chapter V. deals also with periodic transformations, in particular those of period 2 and 3 . I call special attention to the elegant construction of the double points of an involution which is given by two of its pairs.

These are some of the principal features of the introductory chapters. The presentation is elegant, suggestive, and original, excellently adapted for an intelligent student who has already gone through a first introductory course in the theory of functions, such as given, for instance, in Durège, or Burkhardt, or in the function theoretical chapters of Chrystal.

But these chapters would be open to serious objections if they should be intended-as some passages in the Preface seem to indicate-for a beginner, that is a student who is introduced for the first time to the theory of functions. To such a student the many new ideas and new methods of reasoning of the theory of functions, together with the mystery still surrounding the conception of imaginaries, with which he has made only an occasional and mostly very unsatisfactory acquaintance in algebra and calculus, all these things combined usually offer considerable difficulties, and a book intended for the use of beginners ought to make the entrance as easy as possible.

A beginner will hardly be benefited by the delicate and half-philosophical developments of Chapter I. on ordinal numbers; the more so, as they do not give a complete ac- 
count of the matter. In Chapter II. a more systematic, even dry and school-like treatment would better meet his needs, and, for a beginner, the elegant treatment of the bilinear transformation in Chapters III. and V. should be preceded by a more elementary chapter preparing by a study of the simplest special cases: $x+b, a x, 1 / x$ for the most general case.

In Chapter IV. the highly original construction of $\log \xi$ by means of the cone and the thread will puzzle most readers, unless a further explanation be added of what is meant by " winding the thread round the cone without allowing it to slip." But there is a more serious objection to this chapter : Its proofs have only a preliminary character and lay no claim to rigor; and yet some of its results are presupposed in Chapter XIII., where a rigorous analytical theory of the exponential and logarithm is given ; so in particular in $\S 96$ where the tacit assumption is made that the function exp $x$ defined in $\$ 95$ is identical with the function $\exp x$ defined in $\S 32$.

We now turn to the concluding chapters:

Chapter XX. illustrates the construction of Riemann surfaces for algebraic functions by a number of simple examples, which furnish at the same time an opportunity for the distinction between simply and multiply connected surfaces. One paragraph gives an elementary explanation of the fundamental concepts of the theory of groups of linear transformations.

The final Chapter XXII. is devoted to Cauchy's definition of a monogenic function. The necessary and sufficient conditions for the existence of a derivative of a function $u+i v$ of $x+i y$ are given, one of the necessary conditions (which had been overlooked until very recently) being that $u$ and $v$ must have total differentials. A special paragraph gives an excellent critical discussion of the difficulties underlying Cauchy's definition. One of these is of particular interest: the question is raised whether the continuity of the derivative is a necessary consequence of its existence, a question which has in the meantime been settled by Goursat's discovery that Cauchy's theorem can be proved without presupposing the continuity of the derivative.

In connection with the conditions for monogeneity, the potential is introduced, the equipotential problem is explained and solved for some simple cases, and the solution applied to the problem of the conform representation of a straight line upon a polygon. Finally Green's theorem is given and applied to Cauchy's theorem. 
The authors say in the preface: "We had to make a choice between the methods of Cauchy and those of Weierstrass." I must confess that I cannot see the necessity of such exclusiveness. On the contrary, I believe the authors could materially increase the usefulness of their book by working over, in a new edition, the Chapters II.-V., XX., XXII., into an introductory part, adapted in the first place to the needs of the beginner, giving an elementary exposition of Cauchy's theory with emphasis upon conform representation, accepting to a certain extent intuitional proofs. Such an introduction would be the best preparation for Weierstrass's theory ; it would culminate in Cauchy's theorem on Taylor's series, and here the point of junction with Weierstrass would be gained. A critical chapter showing the hidden difficulties of Cauchy's theory and the insufficiency of geometrical proofs would be an excellent link and would prepare for the standpoint of absolute rigor to be adhered to afterwards. For a student will only properly appreciate the stern beauty of Weierstrass's theory, if it has come to him as a salvation out of the doubts and difficulties of the geometrical methods.

\section{Weierstrass's Theory.}

The rest of the book gives a systematic exposition of Weierstrass's theory of functions, excellent in its general arrangement, clear and rigorous in the details, with the exception of some minor flaws, $*$ too few in number and too insignificant to mar the general impression of excellency. We make the following subdivisions:

$A$. The Preparatory Chapters (VI.-IX.).

$B$. The General Theory of Analytic Functions (X.-XII., XIV., XVI.).

* For the benefit of the readers of the "Introduction" I call attention to the following corrigenda and omissions :

(1) P. 91, the quantity $\gamma$ must be assumed to be some value actually taken by $|f(x)|$.

(2) P. 173, the condition for the edges should be

$$
\xi=+\frac{\pi}{2}, \eta \equiv 0 ; \xi=-\frac{\pi}{2}, \eta \equiv 0 \text {. }
$$

(3) P. 179, replace $\gamma$ by "the maximum value of $|f(x)|$ in the closed region $\left(R^{\prime}+D-\delta\right)$."

(4) P. 253, line 4, $\frac{w}{2}$ must not be a period.

(5) P. 270 , line $6,4 \omega_{\mu}, 4 \omega_{\nu}$ are not primitive.

(6) P. 295, line 14, $a_{n}$ is an integral function of $a_{1}, a_{2}, \cdots, a_{n-1}$ and of the $c_{i k}$ 's with real positive coefficients. 
C. Applications to Special Classes of Functions (XIII., XV., XVII.-XIX., XXI.).

\section{A. The Preparatory Chapters.}

They lay the foundation of the theory by an account of the general theory of limits and infinite series and a chapter on rational functions.

In Chapter VI. the principal theorems concerning limits of infinite sequences and continuous functions of one or two real variables are given on the basis of Dedekind's definition of irrationals; the theorem on uniform continuity leads to a discussion of the general problem of uniform convergence to a limit.

The following chapter (VII.) develops the fundamental properties of rational functions of a complex variable and gives the definitions of "limit, continuity, derivative, zero, pole," for the special case of these functions, but in such a manner that they can at once be generalized.

Chapter VIII. treats of the convergence of infinite series with complex terms. Simple tests of convergence are given and the question of association of terms, the distinction between absolutely and conditionally convergent series, and the conversion of a single series into a double series and vice versa, are carefully discussed.

Next follows (Chapter IX.) the subject of uniform convergence of real series, its connection with continuity and an application to real power series, including their behaviour in the frontier points.

Two desiderata must be mentioned with respect to these preparatory chapters : (1) An exhaustive theory of irrationals should be given, and (2) a scientific theory of complex numbers from the standpoint of linear associative algebra. They are two of the cornerstones of Weierstrass's theory and should not be omitted in an account of it otherwise so detailed and complete.

\section{B. The General Theory of Analytic Functions.}

The reader is now thoroughly prepared for the general theory of analytic functions which is developed in the following chapters along the line of Weierstrass's lectures, still with many minor deviations.

Chapter X. treats of the fundamental properties of powerseries, their domain of convergence, the uniformity of their convergence and the criteria of identity of two power series.

The next Chapter XI. on operations with power series is 
based upon Weierstrass's theorem on series of power series. From this theorem (for which in many cases Cauchy's theorem on double sums may be substituted) follow easily the rules for the multiplication and division of power series, Taylor's theorem for power series along with the rules for differentiation of power series and series of power series.

The continuation of a power series (Chapter XII.) is only another aspect of Taylor's theorem, and thus we have reached Weierstrass's definition of an analytic function, viz. a power series together with the totality of its continuations, direct or indirect. Two theorems are necessary to complete the definition : (1) A power series $P\left(x-x_{0}\right)$ and any one of its direct continuations $P\left(x-x_{0} \mid x_{1}\right)$ coincide throughout their common domain of convergence, (2) The series $P\left(x-x_{0}\right)$ is always a direct or indirect continuation of $P\left(x-x_{0} \mid x_{1}\right)$. From the latter theorem follows that, no matter what element of an analytic function we take as starting point, we always obtain the same totality of power series.

Instead of this second theorem, Harkness and Morley use for the same purpose what they call "standard chains" for the continuation of a power series from a point $a$ to a point $b$, that is, a series of points.

$$
a, x_{1}, x_{2}, \cdots, x_{n}, b
$$

such that each point is in the domain of the following as well as the preceding point. The same series of points may then be used for the continuation from $b$ to $a$. But it should be stated and proved that if $P(x-b)$ is a direct or indirect continuation of $P(x-a)$ reached by a non-reversible chain, the same continuation can also be reached by a reversible ("standard") chain.

Frequently it is convenient to think of the continuation of a power series as taking place along a continuous path from $a$ to $b$. The path must be such that it is possible to choose on it a series of points $x_{1}, x_{2}, \cdots, x_{n}$ between $a$ and $b$ such that each point $x_{i+1}$ together with the arc $x_{i} x_{i+1}$ of the curve is contained in the domain of the point $x_{i}$, and it can be proved* that the value reached in $b$ depends only on the path and not on the choice of the intermediate points. Thus a one valued function is defined along the path.

The authors use such paths of continuation later on to prove the theorem that an analytic function is monodromic in a simply connected region which contains no singular point.

\footnotetext{
* See C. Jordan, Cours d' analyse, $2 d$ ed., I., no. 343.
} 
But these geometrical considerations are rather out of harmony with their strictly Weierstrassian surroundings; to make them rigorous it would be necessary to give purely arithmetical definitions of a "continuous path," "circuit," "simply connected region."

The general theory is completed in Chapter XIV., which is devoted to the singular points. The existence of at least one singular point on the circumference of the circle of convergence of a power series is proved and the characteristic difference between essential and non-essential singular points of one valued functions discussed and it is shown how certain classes of one valued functions are characterized by the nature of their singularities: the rational integral, the rational fractional, the transcendental integral, and the transcendental fractional functions.

It is to be regretted that the authors have helped further to increase the lamentable confusion which already exists in our terminology by the introduction of a new term for the concept which occurs most frequently in the theory of functions, viz., the aggregate of properties of a function, necessary and sufficient for its expansion into a power series. It seemed for a while that the term holomorphic, introduced by Briot and Bouquet, had gained general recognition; it was adopted by C. Jordan (1st ed.), by Forsyth, and by Harkness and Morley (Treatise). And now the latest three text-books on the theory of functions abandon it again : Jordan (2d ed.) returns to Cauchy's original "synectic," Burkhardt uses the term "regular," occasionally used by Weierstrass, and Harkness and Morley introduce the term " analytic about a point," which moreover has the disadvantage that the word analytic is already in use-aside from its general meaning-in a different technical sense in Weierstrass's theory.

Chapter XVI. represents a new departure from the ordinary lin as of Weierstrass's theory; it develops Cauchy's theorems on definite integrals on the basis of Weierstrass's concept of an analytic function. For this purpose it is first shown* that the definite integral-considered as a limit of a sum-

* A difficulty of uniform convergence is overlooked in the proof: It is tacitly assumed that for every $\varepsilon$ a $\delta$ exists such that

$$
\left|\frac{\left(x_{\lambda}+h_{\lambda}\right)^{n+1}-x_{\lambda}^{n+1}}{h_{\lambda}}-(n+1) x_{\lambda}{ }^{n}\right|<\varepsilon
$$

as soon as all $\left|h_{\lambda}\right|<\delta$, no matter where $x_{\lambda}$ is taken on the path; that is, uniform differentiability of $x^{n}$ along the path is assumed. It could easily be proved that this condition is always satisfied. 
of the function $x^{n}$, taken from $x_{0}$ to $x_{1}$ along any path $L$ of finite length, has the value

$$
\frac{x_{1}{ }^{n+1}-x_{0}{ }^{n+1}}{n+1} \text {. }
$$

Next it is proved that for the integration of a power series $P(x)=\Sigma a_{n} x^{n}$ integration by terms is allowed, provided the path lies wholly within the circle of convergence. For such a path we have therefore the result

$$
\int_{x_{0}}^{x_{1}}\left(\Sigma a_{n} x^{n}\right) d x=\Sigma a_{n} \frac{x_{1}^{n+1}-x_{0}^{n+1}}{n+1}=\bar{P}\left(x_{1}\right)-\bar{P}\left(x_{0}\right),
$$

if we denote by $\bar{P}(x)$ the indefinite integral of the series $P(x)$. But this difference is Weierstrass's definition of a definite integral for a power series, and thus the two definitions are reconciled.

To extend the result to the case where the path of integration is not wholly contained in the domain of one element, but still a "path of continuation" for the analytic function under consideration, interpolate on the path a finite number of points chosen, as explained above, and apply the previous theorem to each pair of successive points. The element $P(x)$ continued along $L$ furnishes, along $L$, a one valued function $f(x)$; the indefinite integral $\bar{P}(x)$ can be continued along the same path and furnishes another one valued function $F(x)$, and all along $L: \frac{d F(x)}{d x}=f(x)$. The final result is then

$$
\int_{L} f(x) d x=F\left(x_{1}\right)-F\left(x_{0}\right) .
$$

From this point of view, Cauchy's theorem dwindles down almost to a triviality : it is self-evident if the region around which we integrate is contained in the domain of an element of the integrand, and in the general case it is an immediate consequence of the theorem of Article 107. But we must remember that Cauchy's original theorem states much more than in the present connection, since it does not presuppose the expansibility of the function into a power series.

Cauchy's integral and the theorems on residues follow now easily, and a collection of interesting applications to the computation of real definite integrals concludes the chapter. 


\section{Special Classes of Functions.}

The principles of the preceding general theory are illustrated by a series of applications to special classes of functions, which occupy the remaining chapters of the book.

Chapter XIII. gives an analytic theory of the Exponential and Logarithm. The exponential is defined by the series, and the logarithm as its inverse. Special attention is paid to the "chief values" of the logarithm and the allied many valued functions ; they are distinguished by capital initials from the general values, and defined as follows:

$$
\begin{gathered}
-\pi<A m x \leqq \pi \\
\log x=\log |x|+i A m x .
\end{gathered}
$$

The trigonometric functions $\cos x$, sin $x$ are defined by the series and their properties are derived from those of the exponential. The corresponding conform representation problems are studied, and utilized for the investigation of the chief values of the inverse function.

In Chapter XV., after a succinct account of the theory of infinite products based upon the theorem that

$\lim _{n=\infty} \exp x_{1} \cdot \exp x_{2} \cdots \exp x_{n}=\exp \left(\lim _{n=\infty}\left(x_{1}+x_{2}+x_{n}\right)\right)$,

Weierstrass's construction of the most general transcendental integral function with given zeros is developed, with applications to the trigonometric functions and to the $\Gamma$-functions.

In Chapter XVII. Laurent's theorem is proved by means of Cauchy's integral, and applied to a discussion of the isolated singularities of one valued functions, to the expansion of a periodic function into a Fourier series, and to a succinct and elegant treatment of the $\vartheta$-functions: If $g(x)$ denotes the infinite product

$$
g(x)=(1-x)(1-r x)\left(1-r^{2} x\right) \cdots \quad(|r|<1),
$$

then

$$
f(x)=g(x) g\left(\frac{r}{x}\right)
$$

is a one valued analytic function with the zeros

$$
\cdots, \frac{1}{r^{2}}, \frac{1}{r}, 1, r, r^{2}, \cdots
$$

and the only singular points $x=0$ and $x=\infty$. It can be expanded into a Laurent series, and the substitution $r=q^{2}$, $-x=q \exp 2 \pi i v$ transforms $f(x)$ into the fundamental $\vartheta$-function, and the principal properties of the $\vartheta$-functions 
flow easily from the double representation of $f(x)$ by an infinite product and by an infinite series.

'The following two chapters (XVIII., XIX.) develop the theory of elliptic functions, on the whole along the lines of Weierstrass's lectures but with many deviations and simplifications in the detail arrangements. Thus instead of Weierstrass's half-period $\omega_{2}=\omega_{1}+\omega_{3}$, its negative is used so that

$$
\omega_{1}+\omega_{2}+\omega_{8}=0 \text {. }
$$

This change which, it seems, has been simultaneously and independently introduced by Harkness and Morley,* by Study, and by C. Jordan, is an important improvement, since it leads to a complete symmetry with respect to a cyclic permutation of the indices $1,2,3$.

Considerable simplifications are obtained by an extensive use of integration round a period parallelogram ; I mention in particular the proof of the theorem that for every elliptic function $f(u)$ the sum of the zeros is congruent to the sum of the poles, by means of the integral

$$
\int \frac{u f^{\prime}(u)}{f(u)} d u
$$

taken round the period parallelogram.

In Chapter XXI. a sketch of the elementary properties of algebraic functions from the standpoint of Weierstrass is given. It is proved that an irreducible algebraic equation $F(x, y)=0$ defines a single analytic function $y$ of $x$; its singular points are determined, the Puiseux expansions in the vicinity of ordinary branch points are derived and the problems of multiple points of the curve $F=0$ is solved for the simplest case of ordinary double points.

The presentation is throughout clear, and numerous and well selected examples and exercises illustrate the general theorems.

The authors have adopted, for their exposition of the theory of functions, Weierstrass's standard of absolute arithmetical rigor. They insist that the intuitional method is not in itself sufficient for the superstructure of the theory ; and systematically exclude it from all proofs. At the same time a happy conciseness of expression prevents the clumsiness which so frequently accompanies rigorous deductions.

\footnotetext{
* Treatise (1893), p. 301; Study, Sphaerische Trigonometrie and Elliptische Functionen (1893), p. 190 ; C. Jordan, Cours d'Analyse (1894), II., p. 337 .
} 
A wise moderation has been exercised in the selection of topics, and the arrangement of the rich material is pedagogical as well as logical. The concept of the analytic function takes the central place, everything else is subservient to it, either by preparing for it or by illustrating it. By this means, a harmony and equilibrium between the different parts is attained, which impart to the whole in a remarkable degree the character of an organic unity.

Thus the authors have succeeded in producing not only a work of high scientific and pedagogical value but at the same time of a singular beauty and elegance. But there are numerous beauties of detail as well, for which, however, the reader must be referred to the book itself. A certain freshness and originality pervade the whole, even in places where the authors follow along beaten tracks, and give at every turn evidence of the complete mastery of the subject with which the book is written.

University of Chicago,

Oskar Bolza. June 30, 1899.

\section{MoAULAY'S OCTONIONS.}

Octonions. By A. McAulax, M.A. Cambridge, The University Press, $1898 . \quad 8$ vo, $253 \mathrm{pp}$.

THis treatise is a development of Clifford's biquaternions with applications to ordinary space. The starting point of the analysis is quaternions, combined later with methods from Grassman's Ausdehnungslehre.

The development is open to criticism, as a work for beginners, because of its extremely refined formal character. This is perhaps unavoidable because the book was first compiled as a scientific paper, and, as the author says, he did not feel justified in recasting the whole appropriately. Discussions of formal laws and expert reasoning upon terms that are imperfectly defined, relying upon subsequent developments to bring out their full meaning, are not conducive to clearness of apprehension on the part of a learner. Perhaps as good a review, therefore, as can be made of the book, which is indeed an extensive and thorough development of what must prove to be a valuable analysis, is to give a brief and clear exposition of the octonion system.

The octonion is a quantity defined by four numbers called its tensor, scalar, convert, and pitch, and an axis having a fixed position in space. This makes in all eight numbers, upon 